\title{
Coincidence of normal tension glaucoma, progressive sensorineural hearing loss, and elevated antiphosphatidylserine antibodies
}

\author{
S Kremmer, E Kreuzfelder, E Bachor, K Jahnke, J M Selbach, S Seidahmadi
}

Br J Ophthalmol 2004;88:1259-1262. doi: 10.1136/bjo.2003.040832

Background: Recently, in patients with normal tension glaucoma (NTG) elevated levels of antiphosphatidylserine antibodies (APSA), a subgroup of antiphospholipid antibodies (APLA) were found. Progressive sensorineural hearing loss (PSHL) is associated with autoimmune diseases and also the presence of APLA.

Methods: To investigate a possible association between NTG and PSHL, 34 patients (age range 31-81 years) with NTG were evaluated for evidence of audiovestibular disorders. Besides ophthalmological standard examinations (slit lamp, $I O P$, funduscopy, perimetry) scanning laser tomography and polarimetry were performed. From all patients' audiograms, stapedial thresholds and otoacoustic emissions were obtained. The serological testing of patients and controls (40 healthy blood donors older than 50 years) concerned $\lg G$ and lgM levels of antibodies against phosphatidylserine (APSA) and $\beta 2$ glycoprotein.

Results: 23 of 34 NTG patients had hearing loss (PSHL $n=11$; presbyacusis $n=12$ ). The NTG patients had significantly higher APSA levels than controls. Elevated APSA concentrations were significantly more frequent in patients with NTG and hearing loss compared with NTG patients with normacusis.

Conclusions: These findings show that NTG and hearing loss have a high coincidence. The elevated APSA levels may indicate an association with similar systemic autoimmune processes.

1 $\mathrm{n}$ recent years, the understanding of development and progression of glaucomatous optic nerve damage has changed. There is accumulating evidence for a multifactorial pathogenesis of glaucomatous optic neuropathy. Besides an elevated intraocular pressure (IOP), there is special emphasis on cardiovascular and haematological risk factors and also on genetic and immunological aspects. ${ }^{1-6}$ Recently, Kremmer et $a l^{7}$ found elevated levels of a subgroup of antiphospholipid antibodies, antiphosphatidylserine antibodies (APSA), in patients with normal tension glaucoma (NTG) compared to patients with primary open angle glaucoma (POAG) and age matched healthy controls. These findings may be interpreted as a sign for a generalised disease.

Although the pathogenesis of progressive sensorineural hearing loss often remains unclear some research activities have focused on the role of autoantibodies against antigens in the inner ear. This concept was introduced by Lehnhardt ${ }^{8}$ and is supported by the fact that hearing loss is associated with different autoimmune diseases such as Cogan syndrome, rheumatoid arthritis, ${ }^{9}$ Sjögren syndrome, ${ }^{10}$ and Behçet's disease. ${ }^{11}$

Hisashi et $a l^{12}$ were the first to demonstrate an association between progressive sensorineural hearing loss in patients with lupus erythematodes and antiphospholipid antibodies. They proposed that in patients with lupus erythematodes these antibodies are causative for thrombosis of the labyrinth leading to progressive sensorineural hearing loss.

Phospholipids are constituents of all membranes and are divided in many subspecies such as phosphatidylserine. It has been theorised that APSA can be generated by any pathological conditions shifting phosphatidylserine from the inner membrane leaflets to the external membrane leaflets of cells. ${ }^{13}$ This shift is the beginning of the apoptotic mechanism and leads to cell destruction and ischaemia in endothelial cells. ${ }^{14}$ This permits a number of phospholipid binding proteins to be presented to the immune system in unique antigenic conformations, giving rise to antibody production.

Antiphospholipid antibodies were found in patients with autoimmune disease and have been associated with arterial and venous thrombosis, thrombocytopenia, fetal loss, and hearing loss and is summarised as antiphospholipid syndrome. ${ }^{15}$

In this study, we therefore wanted to investigate a possible coincidence between NTG and progressive sensorineural hearing loss (PSHL) and the association to APSA.

\section{PATIENTS AND METHODS}

Thirty four consecutive patients with NTG were included in the study. The average age of the patients was 65 years; 23 patients were female and 11 were male. All gave their consent before their inclusion in the study. Studies were performed in accordance with the ethical standards of the Declaration of Helsinki.

The diagnosis of NTG was established by typical optic disc and visual field damage. In addition, the differentiation between POAG and NTG was based on intraocular pressure (IOP) measurements above (POAG) or below (NTG) $21 \mathrm{~mm} \mathrm{Hg}$. IOP was measured in our hospital for at least 3 days at different times during day and night. Additionally, the hometown ophthalmologists were interviewed for IOP and follow up data of all patients. Ophthalmological standard examinations included slit lamp, Goldmann applanation tonometry, funduscopy, and perimetry. Additionally scanning laser tomography (TopSS, LDT, USA) and polarimetry (GDx, LDT, USA) were performed. NTG patients had mostly moderate visual field defects with absolute arcuate scotomas

Abbreviations: APLA, antiphospholipid antibodies; APSA, antiphosphatidylserine antibodies; IOP, intraocular pressure; NTG normal tension glaucoma; $\mathrm{POAG}$, primary open angle glaucoma; $\mathrm{PSHL}$, progressive sensorineural hearing loss 


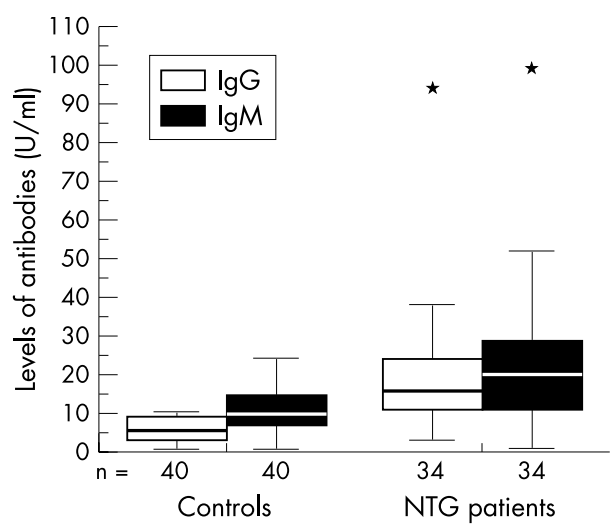

Figure 1 Levels of antiphosphatidylserine $\lg G$ and $\lg M$ antibodies from 34 normal tension glaucoma (NTG) patients and 40 healthy controls. The bars extend from the 25 th percentile to the 75 th percentile with a horizontal line at the median. ${ }^{*} p<0.05 v$ control.

of less than one quadrant (stage 2-3 according to the glaucoma classification of Aulhorn). Visual acuity was better than $20 / 40$.

Additionally, these patients were examined by pure tone audiogram, stapedial thresholds, and transitory otoacoustic emissions. For audiometry a Beomat (Siemens, Erlangen, Germany) and for otoacustic emissions an ILO 88 (Hortman, Leverkusen, Germany) were used.

Hearing loss was classified in low, middle, and high frequency loss and compared to previously established hearing thresholds in patients with normal hearing and presbyacusis. ${ }^{16}{ }^{17}$ Presbyacusis was defined as hearing loss of high frequencies in elderly people ${ }^{16}{ }^{17}$ whereas PSHL was age independent.

As a control group for autoantibody measurements 40 healthy blood donors from the blood bank of the University of Essen (average age 62 years, 23 male and 17 female) were included in our study. They had neither ophthalmological nor otological diseases nor systemic disorders such as arterial or venous thrombosis, thrombocytopenia, autoimmune diseases, or reproductive disorders.

Venous blood samples were drawn from patients and controls and sera obtained after centrifuge. All sera were tested for the presence of antibodies against phosphatidylserine (APSA) of the classes IgM, IgG and against $\beta 2$ glycoprotein using enzyme immunoassays (Imtec, Berlin, Germany). APSA levels of $>15 \mathrm{U} / \mathrm{ml}$ and concentrations of $\beta 2$ glycoprotein antibodies $>5 \mathrm{U} / \mathrm{ml}$ were defined as elevated.

For statistical analysis of different frequencies Fisher's exact test was used. ANOVA with post hoc Dunnet test was used for comparison of antibody concentrations of patients and controls. A p value $<0.05$ was considered as significant.

\section{RESULTS}

APSA concentrations were significantly higher in NTG patients compared to healthy controls (fig l).
Interestingly, the frequency of elevated IgG APSA concentrations in the NTG patients with hearing loss was significantly increased as compared to NTG patients with normacusis and healthy controls (table 1 ).

Twenty three NTG patients (68\%) had hearing loss, mostly affecting the high $(41 \%)$ and the middle frequencies $(32 \%)$. After excluding presbyacusis in $12(35 \%)$ NTG patients, 11 NTG patients $(32 \%)$ had a pathological hearing loss and 11 NTG patients (32\%) had normacusis defined by age matched controls. ${ }^{16}$ Twenty seven NTG patients (79\%) showed reproducible levels of transitory otoacoustic emissions indicating normal outer hair cell function. No reproducible transitory otoacoustic emissions were found in seven patients $(20 \%)$.

Six NTG patients (18\%) had a positive history of thromboembolic disease, four of these patients had a pathological hearing loss and two presbyacusis or normacusis.

In NTG patients, in the group with presbyacusis and normacusis, levels of IgM APSA concentrations were significantly higher $(p<0.05)$ as in healthy controls, whereas no significant differences of IgM APSA concentrations between NTG patients with normacusis, PSHL, and presbyacusis were found (fig 2).

Levels of IgG APSA concentrations were significantly increased in the NTG subgroup with PSHL $(p<0.05)$ compared to healthy controls.

Levels of anti- $\beta 2$ glycoprotein were in the normal range and not significantly different between patients and controls.

\section{DISCUSSION}

In the past few years it was shown that autoimmune phenomena are associated with hearing loss. Naarendorp et $a l^{18}{ }^{19}$ found a correlation between sudden hearing loss and systemic lupus erythematosus. Moreover, progressive hearing loss may be associated with increased autoantibody levels: Tumiati et al ${ }^{10}$ were able to demonstrate an increased

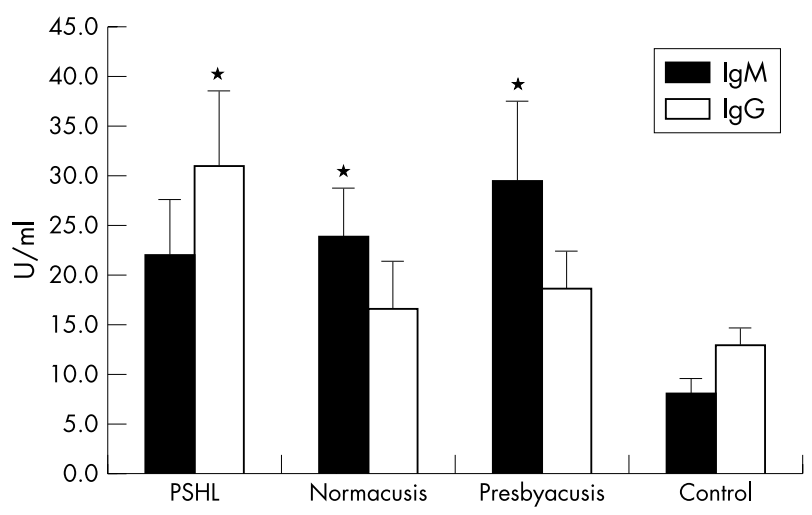

Figure 2 Immunoglobulin (lg) $G$ or $M$ concentrations (SEM) of antiphosphatidylserine antibodies (APSA) in normal tension glaucoma patients with different otoacustic characteristics and in controls; PSHL: progressive sensorineural hearing loss; ${ }^{*} \mathrm{p}<0.05 v$ control.

Table 1 Number of elevated antiphosphatidylserine antibody (APSA) levels in 34 patients with normal tension glaucoma (NTG) and different otoacustic characteristics as well as 40 healthy controls. Fishers exact test

\begin{tabular}{|c|c|c|c|c|}
\hline \multirow[b]{2}{*}{ Immunoglobulin (Ig) class of APSA } & \multicolumn{4}{|c|}{ Number of NTG patients with elevated antibody concentrations } \\
\hline & Presbyacusis $(n=12)$ & PSHL $(n=11)$ & Normacusis $(n=11)$ & Controls $(n=40)$ \\
\hline $\begin{array}{l}\lg G \\
\lg M \\
\lg G+M\end{array}$ & $\begin{array}{l}7(58 \%)^{*} \dagger \\
7(58 \%) \\
4(33 \%)\end{array}$ & $\begin{array}{l}9(82 \%)^{* *} \ddagger \\
7(64 \%)^{*} \ddagger \\
5(45 \%)^{\text {ns }} \neq\end{array}$ & $\begin{array}{l}3(27 \%))^{* *} \S \\
7(64 \%) \\
3(27 \%)\end{array}$ & $\begin{array}{l}3(7.5 \%)^{* *} \\
10(25 \%)^{* *} \\
11(27.5 \%)^{\text {ns }}\end{array}$ \\
\hline
\end{tabular}


concentration of IgG anticardiolipin antibodies in 30\% of patients with Sjögren's syndrome and sensorineural hearing loss.

In principle, two different entities of autoantibodies were found to be associated with glaucoma: autoantibodies against specific proteins of the retina and the optic nerve ${ }^{20-24}$ or against more common antigen $\mathrm{s}^{25-28}$ such as extractable nuclear antigens, small heat shock proteins and serum antibodies against neuron specific enolase.

Interestingly, Shokoohi $e_{t} a^{29}$ reported on elevated antiphospholipid antibodies in NTG and POAG patients. Recently, we observed an increase of antibodies against APSA in NTG patients compared with POAG patients and healthy controls. APSA are a subgroup of antiphospholipid antibodies which are one of the hallmarks of the antiphospholipid syndrome. APSA may be important because of their binding specificity to phosphatidylserine molecules which become accessible during apoptosis, which in turn may lead to local thrombosis.

As elevated concentrations of antibodies against phosphatidylserine were markedly increased in NTG but not in POAG and age matched controls we concluded that these findings are a sign of generalised disease. Therefore, we performed inner ear diagnostic tests in some of these NTG patients and found that $68 \%$ had pathological audiograms (PSHL in $32 \%$ and presbyacusis in 35\%).

It is very difficult to find epidemiological data in the literature on PSHL alone because it has various causes. A survey of the US National Center of Health Statistics, ${ }^{30}$ however, estimated that $14 \%$ of individuals between the ages of 45 and 64 years and 23\% of individuals between 65 and 74 years have hearing loss regardless of the underlying pathology. In an Italian study ${ }^{31}$ based on a sample of 2216 subjects ( $>18$ years) $22 \%$ had sensorineural hearing loss. Most common aetiology was presbyacusis (14.5\%) and progressive vascular disorders $(3.6 \%)$.

Interestingly, a higher prevalence of antiphosphatidylserine antibodies of the IgG class was seen in NTG patients with hearing loss in comparison to NTG patients with normacusis. This finding suggests a similar pathological pathway as a sign for generalised disease.

The increase of the same antibody entity in patients suffering from both, NTG and PSHL may indicate an association with similar systemic autoimmune processes. Interestingly, $35 \%$ of the NTG patients and $45 \%$ of NTG patients with PSHL had both elevated IgG and IgM APSA levels. This may be indicative of an active (IgM) and persistent (IgG) autoimmune process.

Patients with presbyacusis or PSHL showed a significant difference compared to patients with normacusis, suggesting that some patients with presbyacusis also have elevated antiphosphatidylserine antibodies. This is not surprising because antiphospholipid antibodies increase with age. The smaller occurrence of hearing loss compared to normal tension glaucoma might be explained by the fact that the terminal vessel pathway in the eye seems to be more vulnerable to blood supply disturbances or patients are more sensitive to the sense of vision than to hearing. It has been shown that apoptosis can be induced by antiphosphatidylserine antibodies, which results in occlusion of small vessels by thromboemboli and finally leads to disturbance of the microcirculation in the inner ear and eye.

In contrast with APSA, anti- $\beta 2$ glycoprotein antibodies were considered as not important for the pathogenesis of normal tension glaucoma.

Based on our findings all patients with NTG or significant hearing loss and elevated levels of IgG and IgM antibodies against phosphatidylserine should have further ophthalmological or otological work up. In a selected group of patients the inner ear is also involved. Interestingly, Hoyng et $a l^{32}$ found spontaneous platelet aggregation in POAG patients with visual field deterioration more increased as in POAG patients without progressive visual field loss and glaucoma suspects. O'Brien et $a l^{33}$ and Matsumoto et $a l^{34}$ found NTG and, to a lesser extent, POAG associated with increased platelet aggregability and they recommended a treatment with low dose acetylsalicylic acid. Additionally, in other diseases with elevated antiphospholipid antibodies a treatment with coumarin derivates and acetylsalicylic acid was successfully applied. ${ }^{18}{ }^{19}$ Further studies must show if such an anticoagulative treatment in patients with elevated APSA levels and NTG and hearing loss might also be an option.

\section{Authors' affiliations}

S Kremmer, J M Selbach, Department of Ophthalmology, University Hospital Essen, Essen, Germany

E Kreuzfelder, Department of Immunology, University Hospital Essen, Essen, Germany

E Bachor, K Jahnke, S Seidahmadi, Department of

Otorhinolaryngology, University Hospital Essen, Essen, Germany

Correspondence to: Stephan Kremmer, MD, Department of

Ophthalmology, University Hospital Essen, Hufelandstrasse 55, 45122

Essen, Germany; stephan.kremmer@uni-essen.de

Accepted for publication 29 February 2004

\section{References}

1 Drance SM, Sweeney VP, Morgan RW, et al. Studies of factors involved in the production of low tension glaucoma. Arch Ophthalmol 1973:89:457-65.

2 Flammer J, Orgul S, Costa VP, et al. The impact of ocular blood flow in glaucoma. Prog Retin Eye Res 2002;21:359-93.

3 Grunwald JE, Piltz J, Hariprasad SM, et al. Optic nerve blood flow in glaucoma: effect of systemic hypertension. Am J Ophthalmol 1999; 127:516-22.

4 Hayreh SS. The role of age and cardiovascular disease in glaucomatous optic neuropathy. Surv Ophthalmol 1999;43(Suppl 1):S27-42.

5 Kremmer S, Selbach JM, Schäfers RF, et al. Cardiovascular risk profile in the progression of glaucomatous damage. Dt Ärzteb/ 2000;97:A2241-5.

$6 \mathrm{Wax}$ MB. Is there a role for the immune system in glaucomatous optic neuropathy? Curr Opin Ophthalmol 2000;11:145-50.

7 Kremmer S, Kreuzfelder E, Klein R, et al. Antiphosphatidylserine antibodies are elevated in normal tension glaucoma. Clin Exp Immunol $2001 ; 125: 211-15$

8 Lehnhardt E. Plötzliche Hörstörungen, auf beiden Seiten gleichzeitig auftretend oder nacheinander auftretend. Laryngo-Rhino-Otol 1958;37:1-5.

9 Hughes GB, Barna BP, Kinney SE, et al. Clinical diagnosis of immune innerear disease. Laryngoscope 1988;98:251-3.

10 Tumiati B, Casoli P, Parmeggiani A. Hearing loss in the Sjogren syndrome. Ann Intern Med 1997;126:450-3.

11 Eichhorn A, Kirch W. Sicca-Symptomatik und Hörverlust beim Behcet's Syndrom. Dtsch Med Wochenschr 1998;123:663-6.

12 Hisashi K, Komune S, Taira T, et al. Anticardiolipin antibody-induced sudden profound sensorineural hearing loss. Am J Otolaryngol 1993; 14:275-7.

13 Bevers EM, Confurius P, Dekkers DWC, et al. Regulatory mechanisms of transmembrane phospholipid distributions and pathophysiological implications of transbilayer lipid scrambling. Lupus 1998;7(Suppl 2): S126-31.

14 Lockwood CJ, Rand JH. The immunobiology and obstetrical consequences of antiphospholipid antibodies. Obstet Gynecol Surv 1994;49:432-41.

15 Ordi J, Selva A, Monegal F, et al. Anticardiolipin antibodies and dependence of a serum cofactor. A mechanism of thrombosis. J Rheumatol 1993;20:1321-4

16 Lehnhardt, E. Audiometrische Abgrenzung der Altersschwerhörigkeit von der Lärmschädigung des Gehrs. 1977. Mainz, Süddeutsche Eisen- und StahlBerufsgenossenschaft.

17 Schmidt PH. Presbyacusis. International Audiology 1967;1(Suppl): 1-36.

18 Naarendorp M, Spiera H. Sudden sensorineural hearing loss in patients with systemic lupus erythematosus or lupus-like syndromes and antiphospholipid antibodies. J Rheumatol 1998;25:589-92.

19 Laurikainen E, Aantaa E, Kallinen J. Electronystagmographic findings and recovery of cochlear and vestibular function in patients suffering from sudden deafness with a special reference to the effect of anticoagulation. Audiology 1989;28:262-7.

20 Tezel G, Edward DP, Wax MB. Serum autoantibodies to optic nerve head glycosaminoglycans in patients with glaucoma. Arch Ophthalmol 1999; 117:917-24. 
21 Ikeda $\mathrm{Y}$, Ohguro $\mathrm{H}$, Maruyama I. Two cases of primary open angle glaucoma with serum autoantibody against retinal ganglion cells. Jpn J Ophthalmol 2000;44:648-52.

22 Tomita G. The optic nerve head in normal-tension glaucoma. Curr Opin Ophthalmol 2000;11:116-20.

23 Yang J, Tezel G, Patil RV, et al. Serum autoantibody against glutathione S-transferase in patients with glaucoma. Invest Ophthalmol Vis Sci 2001:42:1273-6.

24 Tezel G, Seigel GM, Wax MB. Autoantibodies to small heat shock proteins in glaucoma. Invest Ophthalmol Vis Sci 1998:39:2277-87.

25 Wax MB, Tezel G, Saito I, et al. Anti-Ro/SS-A positivity and heat shock protein antibodies in patients with normal-pressure glaucoma. Am J Ophthalmol 1998;125:145-57.

26 Ikeda Y, Maruyama I, Nakazawa M, et al. Clinical significance of serum antibody against neuron-specific enolase in glaucoma patients. Jpn J Ophthalmol 2002;46:13-17.

27 Maruyama I, Ohguro H, Ikeda Y. Retinal ganglion cells recognized by serum autoantibody against gamma-enolase found in glaucoma patients. Invest Ophthalmol Vis Sci 2000;41:1657-65.
28 Maruyama I, Maeda T, Okisaka S, et al. Autoantibody against neuronspecific enolase found in glaucoma patients causes retinal dysfunction in vivo. Jpn J Ophthalmol 2002;46:1-12.

29 Shokoohi KK, Shin DH, Elliott D, et al. Antiphospholipid antibodies in patients with normal tension glaucoma. Invest Ophthalmol Vis Sci 1999:40(Suppl):342.

30 Benson V, Marano MA. Current estimates from the National Health Interview Survey. Vital Health Stat 1998;10:199.

31 Quaranta A, Assennato G. Causes of hearing loss in Italy. Audiologia Newsletter 1997:3:31-3.

32 Hoyng PF, de Jong $\mathrm{N}$, Oosting $\mathrm{H}$, et al. Platelet aggregation, disc haemorrhage and progressive loss of visual fields in glaucoma. A seven year follow-up study on glaucoma. Int Ophthalmol 1992;16:65-73.

33 O'Brien C, Butt Z, Ludlam C, et al. Activation of the coagulation cascade in untreated primary open angle glaucoma. Ophthalmology 1997; 104:725-9.

34 Matsumoto M, Matsuhashi H, Nakazawa M. Normal tension glaucoma and primary open angle glaucoma associated with increased platelet aggregability. Invest Ophthalmol Vis Sci 1999;40(Suppl):353. 